$1-1-2013$

\title{
Spontaneous Ion Beam Formation In The Laboratory, Space, And Simulation
}

J. Carr

P. A. Cassak

M. Galante

A. M. Keesee

G. Lusk

See next page for additional authors

Follow this and additional works at: https://researchrepository.wvu.edu/faculty_publications

\section{Digital Commons Citation}

Carr, J.; Cassak, P. A.; Galante, M.; Keesee, A. M.; Lusk, G.; Magee, R. M; McCarren, D.; Scime, E. E.; Sears, S.; and Vandervort, R., "Spontaneous Ion Beam Formation In The Laboratory, Space, And Simulation" (2013). Faculty Scholarship. 1035.

https://researchrepository.wvu.edu/faculty_publications/1035 
Authors

J. Carr, P. A. Cassak, M. Galante, A. M. Keesee, G. Lusk, R. M Magee, D. McCarren, E. E. Scime, S. Sears, and R. Vandervort 
Spontaneous ion beam formation in the laboratory, space, and simulation

J. Carr, P. A. Cassak, M. Galante, A. M. Keesee, G. Lusk et al.

Citation: Phys. Plasmas 20, 072118 (2013); doi: 10.1063/1.4817263

View online: http://dx.doi.org/10.1063/1.4817263

View Table of Contents: http://pop.aip.org/resource/1/PHPAEN/v20/i7

Published by the AIP Publishing LLC.

\section{Additional information on Phys. Plasmas}

Journal Homepage: http://pop.aip.org/

Journal Information: http://pop.aip.org/about/about_the_journal

Top downloads: http://pop.aip.org/features/most_downloaded

Information for Authors: http://pop.aip.org/authors

\section{ADVERTISEMENT}

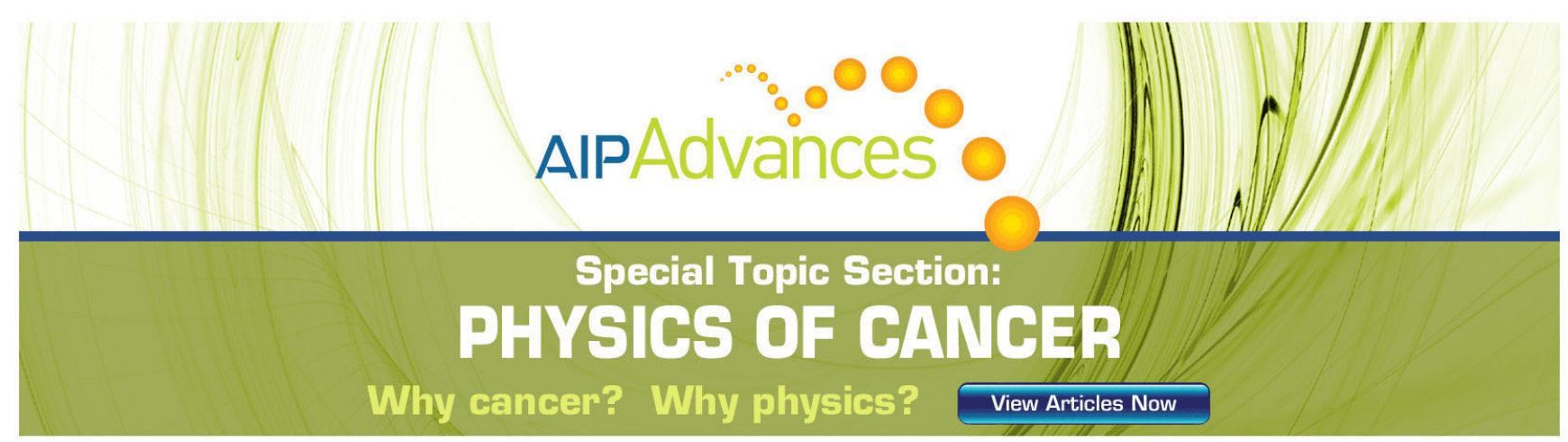




\section{Spontaneous ion beam formation in the laboratory, space, and simulation}

J. Carr, Jr., ${ }^{1}$ P. A. Cassak, ${ }^{1}$ M. Galante,${ }^{1}$ A. M. Keesee, ${ }^{1}$ G. Lusk,${ }^{1}$ R. M. Magee,${ }^{1}$

D. McCarren, ${ }^{1}$ E. E. Scime, ${ }^{1}$ S. Sears, ${ }^{1}$ R. Vandervort, ${ }^{1}$ N. Gulbrandsen, ${ }^{2}$ Martin Goldman, ${ }^{3}$

David Newman, ${ }^{3}$ and J. P. Eastwood ${ }^{4}$

${ }^{1}$ Department of Physics, West Virginia University, Morgantown, West Virginia 26506, USA

${ }^{2}$ University of Troms $\phi$, Troms $\phi$, Norway

${ }^{3}$ Department of Physics, University of Colorado-Boulder, Boulder, Colorado 80309, USA

${ }^{4}$ The Blackett Laboratory, Imperial College London, London SW7 2AZ, United Kingdom

(Received 2 April 2013; accepted 3 July 2013; published online 31 July 2013)

We present experimental evidence for the spontaneous formation of multiple double layers within a single divergent magnetic field structure. Downstream of the divergent magnetic field, multiple accelerated ion populations are observed. The similarity of the accelerated ion populations observed in these laboratory experiments to ion populations observed in the magnetosphere and in numerical simulations suggests that the observation of a complex ion velocity distribution alone is insufficient to distinguish between simple plasma expansion and magnetic reconnection. Further, the effective temperature of the aggregate ion population is significantly larger than the temperatures of the individual ion population components, suggesting that insufficiently resolved measurements could misidentify multiple beam creation as ion heating. Ions accelerated in randomly oriented electric fields that mimic heating would have an ion heating rate dependent on the ion charge and mass that is qualitatively consistent with recent experimental observations of ion heating during magnetic reconnection. ( 2013 AIP Publishing LLC. [http://dx.doi.org/10.1063/1.4817263]

\section{INTRODUCTION}

Since 2002, several laboratory experiments have reported observations of spontaneous formation of current free double layers (DLs) in expanding plasmas with a diverging magnetic field. ${ }^{1-5}$ In its simplest form, a DL consists of two spatially separated charge layers, one positive and one negative. A DL acts very much like a sheath. However, whereas a conventional sheath appears at the surface of an object inserted into the plasma or at the plasma boundary, a DL is a freestanding structure that can appear anywhere within the plasma. ${ }^{6}$ In space and laboratory measurements, evidence for a DL is provided by a population of accelerated ions or direct measurements of the electric potential structure. In the laboratory, laser induced fluorescence (LIF) 3 , or an energy analyzer probe ${ }^{2}$ is employed to measure the entire ion velocity distribution function (ivdf), confirming the existence of a DL by detecting the ion beam. In ivdf measurements by spacecraft, DLs have been identified in magnetospheric regions ranging from the auroral zone to the plasma sheet. $^{9}$

In this work, we present the first spatially localized observations of the spontaneous formation of multiple DL regions in a simple, current-free, divergent magnetic field. We show that the ivdfs measured in these experiments bear a striking similarity to those observed in other experiments (in which multiple DLs are intentionally created) and in exhaust regions of magnetic reconnection events in simulations and in space. While previously it was assumed that the creation of an ivdf comprised of multiple ion beams requires a complex electric field structure normally associated with magnetic reconnection, we suggest that complex ivdfs can arise from nothing more than an expanding magnetic field. This is potentially important for the accurate identification of reconnection events from satellites that measure distribution functions but not magnetic fields. We also show that the measured ivdfs are sufficiently broad that the average kinetic energy in the frame of the average flow, the "effective temperature," is an order of magnitude larger than the ion temperature upstream of the divergent magnetic field even though individual ion beams do not display significant heating. As a result, in satellite observations or experiments, underresolving ivdfs could lead to a false identification of ion beam creation as ion heating.

\section{EXPERIMENTAL APPARATUS}

The experiments reported here are performed in the Hot hELIcon eXperiment, ${ }^{10}$ comprised of a $61 \mathrm{~cm}$ long, $10 \mathrm{~cm}$ diameter, Pyrex ${ }^{\mathrm{TM}}$ source chamber connected to a $91 \mathrm{~cm}$ long, $15 \mathrm{~cm}$ diameter stainless steel chamber. The stainless steel chamber opens into a $2 \mathrm{~m}$ diameter, $4.5 \mathrm{~m}$ long expansion chamber. The magnetic field ranges from 0 to $1300 \mathrm{G}$ in the source and $0-150 \mathrm{G}$ in the expansion chamber. Argon plasmas are created at neutral pressures in the source ranging from 0.1 to $100 \mathrm{mTorr}$. Rf power of up to $2.0 \mathrm{~kW}$ over a frequency range of $6-18 \mathrm{MHz}$ is coupled into a $19 \mathrm{~cm}$ half wave, helical antenna to create the steady state plasma. An important feature for these experiments is that the large pumping rate in the expansion chamber $(3200 \mathrm{l} / \mathrm{s})$ results in a downstream neutral pressure ten times smaller than in the source. Previous measurements in this system have also shown that the neutral pressure profile is hollow, with the on-axis pressure as small as one-tenth the edge neutral pressure. ${ }^{11}$ Thus, the mean free path for ion-neutral charge exchange for these plasmas is many tens of centimeters. Characteristic electron temperature and densities in HELIX 
for these experimental parameters are $T_{e}=5.8 \mathrm{eV}$ and $n=2 \times 10^{12} \mathrm{~cm}^{-3}$.

The argon ivdf is measured non-perturbatively through velocity resolved LIF of the ions. Each LIF measurement (see Ref. 12 for a complete description of the diagnostic system) consists of sweeping the frequency of the very narrow bandwidth laser through the thermally broadened ion velocity distribution function. Measurement of the intensity of the photon emission from the excited state as a function of laser frequency provides a direct measurement of the ivdf. As the laser frequency is swept over as much as $60 \mathrm{GHz}$, the fluorescent emission from the pumped excited state is collected with a reentrant glass probe in the expansion chamber and transported to a filtered photomultiplier detector.

\section{LIF MEASUREMENTS OF MULTIPLE ION BEAMS}

Shown in Figure 1(a) is a typical, "no-beam," LIF measurement of the ivdf in the expansion region for an operating
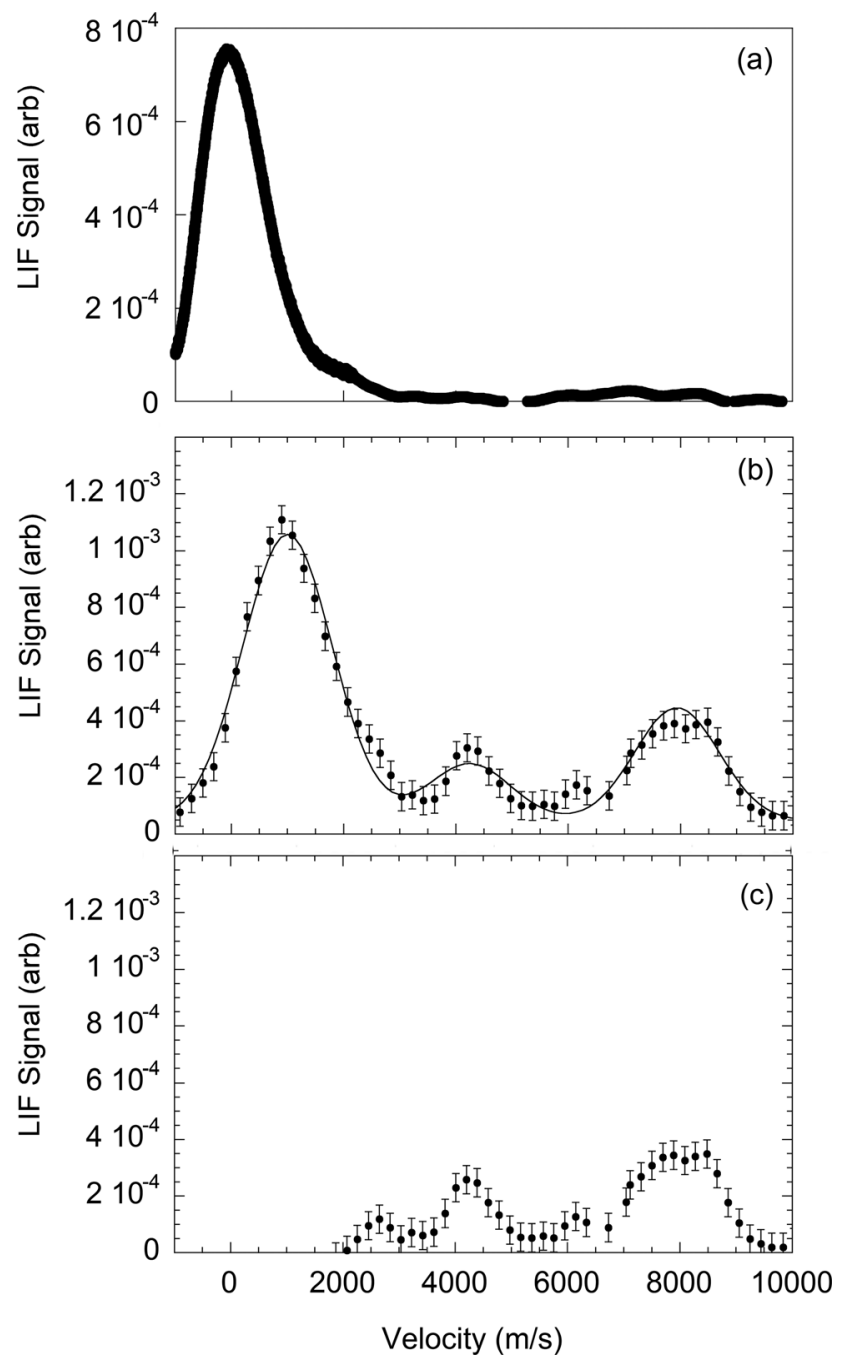

FIG. 1. (a) Typical LIF measured ivdf (filled circles) as a function of velocity in the expansion chamber for operating parameters that do not result in the formation of an ion beam. (b) LIF measured ivdf (circles) as a function of velocity in the expansion chamber $38 \mathrm{~cm}$ downstream of the plasma source. A three Maxwellian component fit (solid line) yields identical ion temperatures of $\sim 0.16 \mathrm{eV}$ for all three components. (c) Same data as (b) minus the fit to the stationary background population. A very small third accelerated population appears around $2500 \mathrm{~m} / \mathrm{s}$. pressure of 2.0 mTorr, an upstream magnetic field of $1000 \mathrm{G}$, and a downstream magnetic field of $38 \mathrm{G}$. These source parameters, particularly the neutral pressure of 2.0 mTorr, do not result in formation of a DL in the expansion chamber. Shown in Figure 1(b) is an LIF measurement of the ivdf for a lower operating pressure of $1.8 \mathrm{mTorr}$, a driving frequency of $9 \mathrm{MHz}$, an upstream magnetic field of $700 \mathrm{G}$, and a downstream magnetic field of $19 \mathrm{G}$. These measurements were obtained $38 \mathrm{~cm}$ downstream of the plasma source and expansion chamber junction. Three ion populations are evident: a low speed "bulk" population and two ion "beam" populations. The ion gyroradius in the source is approximately $0.5 \mathrm{~cm}$ and $13 \mathrm{~cm}$ in the expansion chamber. Operating pressures of $1.8 \mathrm{mT}$ Torr are atypically low for this experimental facility and stable, steady-state, operation of the plasma source requires large levels of total $\mathrm{rf}$ power $(800 \mathrm{~W}$, measured at the output of the rf amplifier) and careful minimization of the reflected rf power (less than $50 \mathrm{~W}$, measured at the input to the matching network). As noted earlier, spontaneous appearance of a single ion beam in these expanding plasmas has been reported for over a decade (see Figure 2 for examples of such ivdf measurements). That multiple ion beams may spontaneously appear is a new result.

The LIF-measured ion population upstream of the expansion region is well described by a single Maxwellian velocity distribution with a bulk ion temperature of $0.2 \mathrm{eV}$ and a bulk flow towards the expansion region of $\sim 300 \mathrm{~m} / \mathrm{s}$. Previous studies demonstrated that a DL forms at the junction between the source and the expansion chamber. The DL is localized to the region of the strongest magnetic field gradient where the density gradient is also largest. ${ }^{3}$ The highest energy population evident in Figure 1(b), with a flow speed of $\sim 8000 \mathrm{~m} / \mathrm{s}$, corresponds to an argon beam energy of $\sim 13 \mathrm{eV}$. Based on Langmuir probe measurements, the electron temperature in the source is $\sim 6 \mathrm{eV}$. Since the DL potential energy is roughly twice the electron temperature, these DLs are what are commonly called "weak" DLs. The second accelerated ion population that appears around $\sim 4200 \mathrm{~m} / \mathrm{s}$ corresponds to an accelerating potential of $\sim 4 \mathrm{~V}$. An enhanced flux of ions in a narrow energy band is consistent with observations of ion beams in space plasmas and is typically attributed to DLs ${ }^{13}$ (whereas fluxes over a broad band

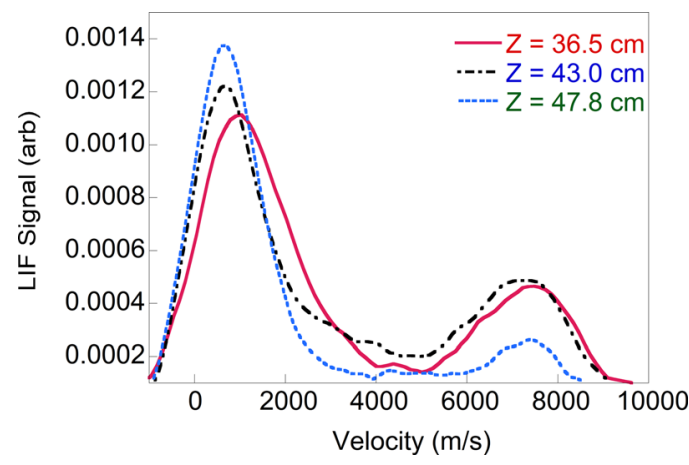

FIG. 2. Smoothed fits to LIF measured ivdfs for a single double layer case as a function of velocity in the expansion chamber at three different downstream locations for source parameters that result in the formation of only a single downstream ion beam population. Over more than $11 \mathrm{~cm}$, there is no change in the velocity of the accelerated ion population. 
of energy are typically attributed to stochastic acceleration in the electric fields arising from turbulent wave activity ${ }^{14}$ ).

Additional LIF measurements along the axis of the experiment for operating conditions that result in formation of only a single beam (a smaller magnetic field expansion ratio and lower rf power levels) show that while the intensity of the LIF signal decreases with distance from the acceleration region (consistent with quenching of the metastable argon ion state by electron-ion collisions as seen in previous experiments ${ }^{1,15}$ ), the velocities of accelerated ion peaks in ivdf measurements are unchanged with distance (over 10s of $\mathrm{cm}$ ) from the acceleration region (see Figure 2). Although collisions with background electrons do depopulate the initial metastable state necessary for the LIF measurement, the momentum loss to the electrons and any background neutrals is negligible over the distances of these measurements. The persistence of the beam energies downstream of the DL is also independently confirmed with retarding field energy analyzer (RFEA) ${ }^{16}$ measurements. The axially resolved LIF measurements shown in Figure 2 confirm that the ion beams are created upstream of the measurement location and can travel for tens of centimeters without significant degradation in beam energy.

To understand how it is possible for ion beams of different energies to appear at a single downstream measurement location, we note that this is not the first time multiple accelerated ion populations have been observed in a helicon plasma. In a series of experiments on the Magnetized Nozzle eXperiment, multiple double layers were intentionally created by introducing grounded apertures upstream from a magnetic nozzle. ${ }^{17}$ Axially resolved LIF measurements demonstrated that ions accelerated in the sheath formed by the aperture traveled nearly $30 \mathrm{~cm}$ to the magnetic nozzle where they were further accelerated by the double layer at the magnetic nozzle. Between the grounded aperture and the magnetic nozzle, a single accelerated ion population and the background ions created beyond the aperture were both observed with LIF. Downstream of the second double layer, three distinct ion populations were evident: doubly accelerated ions, an accelerated ion population comprised of the ions created between the aperture and the magnetic nozzle that were then accelerated by only the double layer at the nozzle, and background ions. Thus, those earlier experiments demonstrated that multiple ion beams will appear in ivdfs when additional ionization occurs between accelerating structures, i.e., new ions are created throughout the experiment by electron impact ionization and those created between the DLs are accelerated downstream by only the second DL (and electrons accelerated into the region between the DLs also provide additional ionization ${ }^{7}$ ). The important distinction between the experimental results reported here and the earlier experiments is that here, the multiple ion beams spontaneously appear in a simple divergent magnetic field.

\section{COMPARISON WITH THEORY, SPACE MEASUREMENTS, AND SIMULATIONS}

These experimental results are consistent with a number of theoretical predictions. Over forty years ago, one dimensional particle-in-cell (PIC) simulations of ion acoustic shocks in the region of a strong density gradient predicted the formation of DLs at the location of the density gradient and, if the density gradient was steep enough, the formation of wave-like perturbations in density and plasma potential upstream of the shock. ${ }^{18}$ Such a wave-like potential structure could explain these observations. Although more recent PIC simulations have typically yielded only solitary DL structures, ${ }^{19}$ the review by Singh $^{20}$ suggests that when perpendicular electric fields develop near density gradients due to differing electron and ion Larmor radii, the perpendicular electric fields are shorted out by conducting boundaries in a laboratory plasma. The resulting parallel electric field may then be localized at a single DL or be spread out across multiple DLs. Another very recent theoretical study of the expansion of a collisionless plasma into a plasma of lower density predicts formation of what the authors call "multi-modal" ivdfs as a result of wave breaking phenomena along the expansion interface. ${ }^{21}$

It is also possible that the electric field structures responsible for these observations are simply radially separated structures and that the finite gyroradii of the ions enables different ions to sample different radially localized acceleration regions. Unfortunately, we are unable to access the last few centimeters of the helicon source before the expansion chamber to perform LIF measurements of the axial flow at different radial locations near the end of the helicon source. Such measurements are planned in future experiments. However, RFEA measurements of the ion energy distribution function at different radial locations indicate that the acceleration regions are quite broad radially and therefore support the interpretation that these measurements are indicative of discrete, axially separated, acceleration regions.

Because the ion beams are relatively cold, it is possible to isolate the beams from the bulk ion population. Maxwellian fits (shown in Figure 1(b)) to each of the three well-defined populations yield identical ion temperatures of $\sim 0.15 \mathrm{eV}$. As noted previously, the large "bulk" ion population is the locally created "downstream" plasma. ${ }^{3}$ In Figure 1(c), the background ion population in Figure 1(b) has been subtracted from the full measurement to highlight just the portion of the ivdf accelerated by the DL region. After the subtraction, it is clear that there are at least three accelerated ion populations, not just two.

The spontaneous formation of electrostatic structures capable of generating multiple accelerated ion populations in a simple expanding magnetic field is a remarkable and new result. The mere possibility that such simple magnetic geometries are capable of producing complex ivdfs has important implications for the interpretation of ivdf measurements in all plasmas. For example, complex ivdfs seen in space are often assumed to be a signature of magnetic reconnection. Shown in Figure 3 are two ivdf measurements obtained by the THEMIS spacecraft during a bursty bulk flow event on 26 February 2008. Details of the THEMIS spacecraft and the ion distribution measurement process are discussed in Ref. 22. The THEMIS measurements are shown as a function of velocity along the bulk flow direction. At zero velocity, the THEMIS measurements are contaminated with a large background signal due to photoemission and spacecraft charging. 


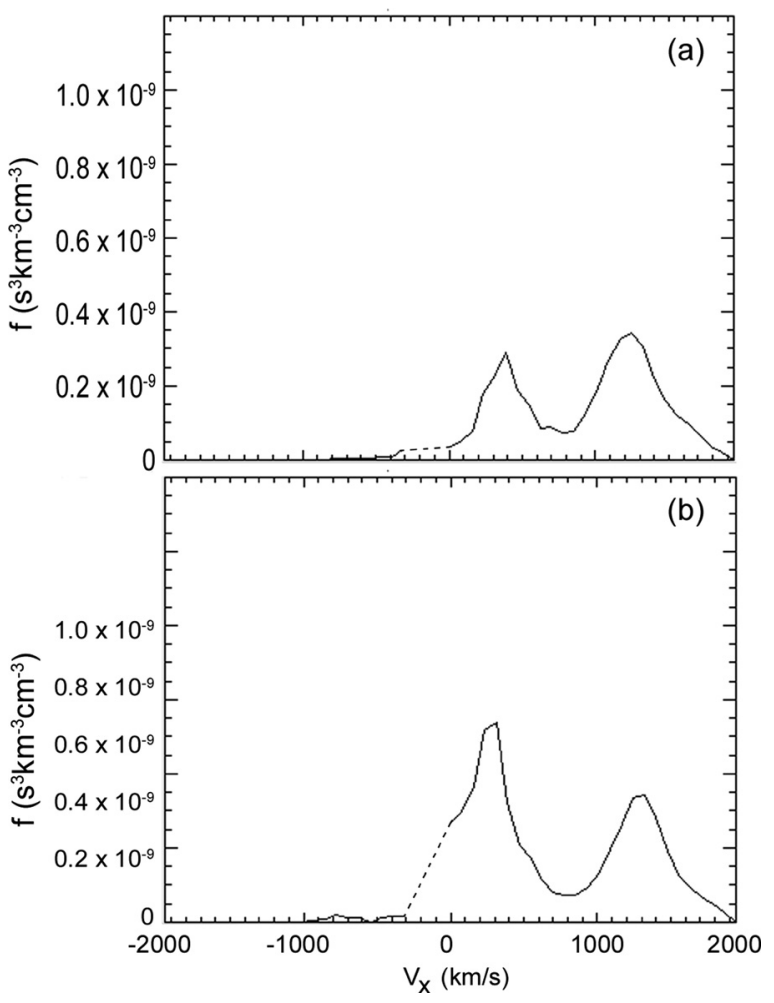

FIG. 3. The ion velocity distribution function along the outflow direction (reduced to one dimension by integrating over the over two velocity components) for a bursty bulk flow event on 26 February 2008 at (a) 11:12:52 and $3 \mathrm{~s}$ later at (b) 11:12:55. A large background signal in the measurement at zero velocity due to photoemission and spacecraft charging has been deleted from the figure (the dashed line corresponds to the contaminated region of the distribution). Two accelerated ion populations appear in both measurements.

Therefore, the low energy portion of the distribution has been removed in Figure 3 and replaced with a dashed line, isolating the ion beams as was done with the laboratory measurements. In both THEMIS measurements, two accelerated ion populations are clearly visible. As in Figure 1, the accelerated ion populations are superthermal (their flow speeds are much greater than their thermal speeds). Similar complex ion velocity distributions are observed routinely by THEMIS, i.e., the data shown in Figure 3 are by no means unique or exceptional.

Complex ivdfs with structures similar to our laboratory observations are also commonly observed in simulations. An ivdf from an implicit two-and-a-half dimensional PIC simulation of magnetic reconnection is shown in Figure 4. The simulation includes a guide field (equal in strength to the reconnecting field) and the computational domain size is $L_{x} \times L_{y}=40 d_{\mathrm{i}} \times 20 d_{\mathrm{i}}$ (where $d_{\mathrm{i}}=c / \omega_{\mathrm{pi}}$ is the ion inertial length, $c$ is the speed of light, $\omega_{p i}=\sqrt{4 \pi n e^{2} / m_{i}}$ is the ion plasma frequency, and $e$ is the electron charge). Periodic boundary conditions are assumed in the $x$ direction and perfect electric conductor boundaries are set at $y=0$ and $y=L_{y}$. The reconnection simulation starts with a classic Harris sheet $^{23}$ of high density particles surrounded by background particles with a density an order of magnitude lower and a magnetic field profile given by a hyperbolic tangent function. Additional details about the simulation and physics assumptions inherent in the model are discussed in Ref. 24. The $x$ direction is along the bulk outflow direction and the

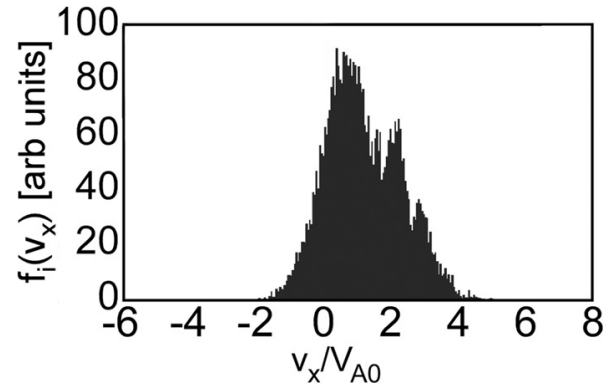

FIG. 4. Ion velocity distribution (as a function of velocity normalized to the initial Alfvén velocity) along the outflow direction from a particle-in-cell numerical simulation of magnetic reconnection. The distribution is obtained 20 ion inertial lengths downstream of the reconnection site and is integrated over the other two coordinate directions. The ion velocity distribution includes two accelerated ion populations plus a stationary background population.

distribution has been integrated over the direction perpendicular to the bulk flow. The velocities are normalized to the reference Alfvén speed $\mathrm{v}_{\mathrm{A}}=B_{0} /\left(\mu_{0} n_{i o} m_{i}\right)^{1 / 2}$, where $B_{0}$ is the background magnetic field strength, $m_{i}$ is the ion mass, and $n_{i o}$ is the initial maximum Harris-sheet ion density. The simulation distribution function is sampled at $20 d_{\mathrm{i}}$ downstream of the reconnection site along the outflow axis many ion cyclotron periods after the onset of reconnection. As in the laboratory and space measurements, multiple accelerated ion populations appear in the ivdf (as well as a background ion population). The total ion distribution at this location includes one population crossing the separatrix without sampling the Hall electric field near the X-line and other populations that have been accelerated by discrete Hall electric field structures that appear near the X-line and along the separatrices. ${ }^{25}$ Further downstream, the populations merge into a single, broad, "hot," ivdf.

\section{DISCUSSION}

Clearly, the appearance of complex ivdfs can be a signature of acceleration in discrete electric field structures that arise during magnetic reconnection. However, based on these new laboratory observations, we conclude that the mere existence of a complex ivdf is not sufficient to posit the existence of magnetic reconnection. Such ivdfs could simply result from plasma expansion and production in a divergent magnetic field (or flows of different source plasmas into a common divergent field region).

Consider the rapid and intense ion heating typically associated with magnetic reconnection in laboratory experiments. ${ }^{26}$ The heating occurs more rapidly than any collisional or viscous timescale in the plasma and the "heated" ion population is well described by a high temperature Maxwellian distribution plus a power-law high-energy tail. ${ }^{27}$ How the ions are heated so rapidly remains an open question in reconnection dominated experiments. However, nearly all such heating measurements are fundamentally unable to resolve structure within the ivdf at the spatial scale of a reconnection layer. Either the spatial resolution of the diagnostic technique exceeded the reconnection scale, the technique employed line integrated measurements, or the velocity resolution of the technique was insufficient to differentiate between complex structure and a broad, hot, velocity distribution. ${ }^{12,28}$ Even 
diagnostic methods with spatial resolution comparable to reconnection layer scale still average over large spatial regions because of the large ion gyroradius of the heavy impurity species used for the measurement. ${ }^{29}$ Therefore, although broad ivdfs are usually interpreted to be indicative of ion heating, typical observations are equally consistent with unresolved ivdfs containing multiple accelerated ion populations. ${ }^{30,31}$

As an example of how the presence of ion beams could be misinterpreted as ion heating, consider that a large fraction of the ion temperatures reported by space-based instruments are based on a straightforward and automatic calculation of the 2nd moment of the entire measured ivdf. Although the LIF-measured temperature of each ion beam population in Figure $1(\mathrm{~b})$ is $0.16 \mathrm{eV}$, the average kinetic energy in the frame of the flow, i.e., the 2nd moment of the ivdf, yields an ion temperature of well over $1 \mathrm{eV}$. Thus, similar naïve analysis of the ivdf shown in Figure 1(b) would conclude that the downstream ion temperature was an order of magnitude hotter than the upstream temperature, even though no significant heating of the ions actually occurred. It is only because THEMIS, with its very high time resolution ion instrument, is capable of resolving relatively small spatial scales that the ion beams are resolvable in the data of Figure 3.

If we assume that ion dynamics during phenomena such as magnetic reconnection are not dominated by thermal processes or wave-particle interactions, but instead result from reversible acceleration in discrete electric fields (as has been shown to occur for single test particles in simulations ${ }^{32}$ ) of varying magnitude and orientation, the rate of ion energization (heating) should depend solely on the energy gained by ions falling through such electric fields: $\Delta\left(m \mathrm{v}^{2} / 2\right) / \Delta t$ $\approx \sqrt{d q^{3} E^{3} / 2 m}$, where $E$ is the electric field in the DL of thickness $d$ and the heating rate is determined by the transit time of the ions in the DL. In other words, the energization rate of ions of different charge-to-mass $(\mathrm{q} / \mathrm{m})$ ratios should scale as $\left(q^{3} / m\right)^{1 / 2}$ and should be independent of the magnitude of the magnetic fluctuation amplitude.

In the Madison Symmetric Torus (MST), the ion temperature during magnetic reconnection doubles or triples in less than $10 \mu \mathrm{s}$. Heating models based on cyclotron heating from magnetic fluctuations and viscous damping of reconnection flows have been proposed. Yet, two decades of measurements have failed to find any significant correlation between ion heating levels and the levels of magnetic fluctuations (levels of ion heating have been shown to correlate with the overall change in stored magnetic energy during magnetic reconnection ${ }^{33}$ ). Recently, the MST experiment reported that their first studies of ion heating for impurity ions of different charge-to-mass ratios demonstrated that the parallel ion heating rate depends on the $(q / m)$ ratio during magnetic reconnection. ${ }^{34}$ However, the overall $(\mathrm{q} / \mathrm{m})$ ratio range was too limited to distinguish between $\left(\mathrm{q}^{3} / \mathrm{m}\right)^{1 / 2}$ or linear $(\mathrm{q} / \mathrm{m})$ scaling. We hypothesize that ions passing through a multitude of tightly packed and randomly oriented reconnection sites with a distribution of total energization "strengths" might rapidly acquire a distribution of three-dimensional velocities that appear to result from a single hot nearMaxwellian parent distribution.
In summary, these new experimental results demonstrate that there is no significant difference in ivdfs arising from reconnection or a simple divergent magnetic field. Our results show that one cannot identify complex ivdfs with reconnection in the absence of magnetic field measurements. More broadly, we emphasize that "hot" ion distribution functions that are under-resolved could actually be ion beams, such as those from randomly oriented electric fields.

\section{ACKNOWLEDGMENTS}

This work was supported by NSF Award No. PHY0611571. J.C., Jr. was supported by WV Space Grant and SREB graduate fellowships. We acknowledge NASA Contract No. NAS5-02099 and V. Angelopoulos for use of data from the THEMIS Mission, specifically C. W. Carlson and J. P. McFadden for use of ESA data. J.P.E. received support from the STFC (Grant No. ST/G00725X/1) at ICL. Work at the University of Colorado was supported by NASA MMS Grant No. NNX08AO84G.

${ }^{1}$ S. A. Cohen, N. S. Siefert, S. Stange, R. F. Boivin, E. E. Scime, and F. M. Levinton, Phys. Plasmas 10, 2593 (2003).

${ }^{2}$ C. Charles and R. Boswell, Appl. Phys. Lett. 82, 1356 (2003).

${ }^{3}$ X. Sun, A. M. Keesee, C. Biloiu, E. E. Scime, A. Meige, C. Charles, and R. Boswell, Phys. Rev. Lett. 95, 025004 (2005).

${ }^{4}$ N. Plihon, C. S. Corr, and P. Chabert, Appl. Phys. Lett. 86, 091501 (2005).

${ }^{5}$ K. Takahashi, C. Charles, R. Bowell, and R. Hatakeyama, Phys. Plasmas 15, 074505 (2008).

${ }^{6}$ N. Hershkowitz, IEEE Trans. Plasma Sci. 22, 11 (1994).

${ }^{7}$ S. Chakraborty Thakur, Z. Harvey, I. A. Biloiu, A. Hansen, R. A. Hardin, W. S. Przybysz, and E. E. Scime, Phys. Rev. Lett. 102, 035004 (2009).

${ }^{8}$ E. E. Scime, I. A. Biloiu, J. Carr, Jr., S. Chakraborty Thakur, M. Galante, A. Hansen, S. Houshmandyar, A. M. Keesee, D. McCarren, S. Sears, C. Biloiu, and X. Sun, Phys. Plasmas 17, 055701 (2010).

${ }^{9}$ R. E. Ergun, L. Andersson, J. Tao, V. Angelopoulos, J. Bonnell, J. P. McFadden, D. E. Larson, S. Eriksson, T. Johansson, C. M. Cully, D. N. Newman, M. V. Goldman, A. Roux, O. LeContel, K.-H. Glassmeier, and W. Baumjohann, Phys. Rev. Lett. 102, 155002 (2009).

${ }^{10}$ P. A. Keiter, E. E. Scime, and M. M. Balkey, Phys. Plasmas 4, 2741 (1997).

${ }^{11}$ A. M. Keesee and E. E. Scime, Plasma Sources Sci. Technol. 16, 742-749 (2007).

${ }^{12}$ A. K. Hansen, M. Galante, D. McCarren, S. Sears, and E. E. Scime, Rev. Sci. Instrum. 81, 10D701 (2010).

${ }^{13}$ J. Birn, A. V. Artemyev, D. N. Baker, M. Echim, M. Hoshino, and L. M. Zelenyi, Space Sci. Rev. 173, 49-102 (2012).

${ }^{14}$ B. A. Bryant, R. Bingham, and U. Deangelis, Phys. Rev. Lett. 68, 37 (1992).

${ }^{15}$ F. Skiff, G. Bachet, and F. Doveil, Phys. Plasmas 8, 3139 (2001).

${ }^{16}$ N. Gulbrandsen, W. J. Miloch, and A. Fredriksen, Contrib. Plasma Phys. 53, 27 (2013).

${ }^{17}$ X. Sun, S. Cohen, and E. E. Scime, Phys. Plasmas 12, 103509 (2005).

${ }^{18}$ R. J. Mason, Phys. Fluids 14, 1943 (1971).

${ }^{19}$ A. Meige, R. W. Boswell, C. Charles, and M. M. Turner, Phys. Plasmas 12, 052317 (2005).

${ }^{20}$ N. Singh, Phys. Plasmas 18, 122105 (2011).

${ }^{21}$ M. Perego, P. D. Howell, M. D. Gunzburger, J. R. Ockendon, and J. E. Allen, Phys. Plasmas 20, 052101 (2013).

${ }^{22}$ J. P. McFadden, C. W. Carlson, D. Larson, M. Ludlam, R. Abiad, B. Elliott, P. Turin, M. Marckwordt, and V. Angelopoulos, Space Sci. Rev. 141, 277 (2008).

${ }^{23}$ E. G. Harris, Nuovo Cimento 23, 115 (1962).

${ }^{24}$ A. Divin, G. Lapenta, S. Markidis, D. L. Newman, and M. V. Goldman, Phys. Plasmas 19, 042110 (2012).

${ }^{25}$ M. A. Shay, J. F. Drake, R. E. Denton, and D. Biskamp, J. Geophys. Res. 103, 9165, doi:10.1029/97JA03528 (1998).

${ }^{26}$ E. Scime, S. Hokin, N. Mattor, and C. Watts, Phys. Rev. Lett. 68, 2165 (1992). 
${ }^{27}$ R. M. Magee, D. J. Den Hartog, S. T. A. Kumar, A. F. Almagri, B. E. Chapman, G. Fiksel, V. V. Mirnov, E. D. Mezonlin, and J. B. Titus, Phys. Rev. Lett. 107, 065005 (2011).

${ }^{28}$ V. H. Chaplin, M. Brown, D. H. Cohen, T. Gray, and C. D. Cothran, Phys. Plasmas 16, 042505 (2009).

${ }^{29}$ M. R. Brown, C. D. Cothran, T. Gray, C. E. Myers, and E. V. Belova, Phys. Plasmas 19, 080704 (2012).

${ }^{30}$ T. Intrator, J. Menard, and N. Hershkowitz, Phys. Fluids B 5, 806 (1993).
${ }^{31}$ R. L. Merlino and J. J. Loomis, Phys. Fluids B 2, 2865 (1990).

${ }^{32}$ J. F. Drake, M. Swisdak, T. D. Phan, P. A. Cassak, M. A. Shay, S. T. Lepri, R. P. Lin, E. Quataert, and T. H. Zurbuchen, J. Geophys. Res. 114, A05111, doi:10.1029/2008JA013701 (2009).

${ }^{33}$ S. Gangadhara, D. Craig, D. A. Ennis, D. J. Den Hartog, G. Fiksel, and S. C. Prager, Phys. Plasmas 15, 056121 (2008).

${ }^{34}$ S. T. A. Kumar, D. J. Den Hartog, K. J. Caspary, R. M. Magee, V. V. Mirnov, B. E. Chapman, D. Craig, G. Fiksel, and J. S. Sarff, Phys. Rev. Lett. 108, 125006 (2012). 